This is the final peer-reviewed accepted manuscript of:

Berra, A., Cecchini, S., Cindolo, F., Ferdinando, D.D., Jollet, C., Longhin, A., Ludovici, L., Mandrioli, G., Mauri, N., Meregaglia, A., Paoloni, A., Pasqualini, L., Patrizii, L., Pozzato, M., Pupilli, F., Prest, M., Sirri, G., Terranova, F., Vallazza, E., Votano, L., Longitudinally segmented shashlik calorimeters with SiPM readout. Nuclear Instruments and Methods in Physics Research, Section A: Accelerators, Spectrometers, Detectors and Associated Equipment, Volume 845 (2017), 511-514.

The final published version is available online at:

https://doi.org/10.1016/j.nima.2016.04.025

(C) 2017. This manuscript version is made available under the Creative Commons AttributionNonCommercial-NoDerivs (CC BY-NC-ND) 4.0 International License

(http://creativecommons.org/licenses/by-nc-nd/4.0/) 


\title{
Longitudinally segmented shashlik calorimeters with SiPM readout
}

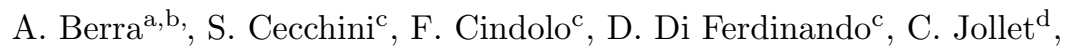 \\ A. Longhin ${ }^{\mathrm{e}}$, L. Ludovici $^{\mathrm{f}}$, G. Mandrioli ${ }^{\mathrm{c}}$, N. Mauri ${ }^{\mathrm{c}}$, A. Meregaglia $^{\mathrm{d}}$,

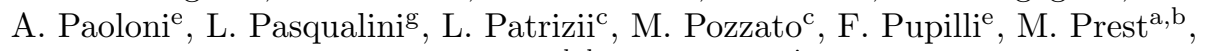

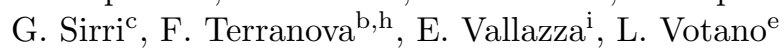 \\ ${ }^{a}$ Università degli Studi dell'Insubria, Via Valleggio, 11 - 22100 Como, Italy \\ ${ }^{b}$ INFN sezione di Milano Bicocca, Piazza della Scienza, 3 - 20126 Milano, Italy \\ ${ }^{c}$ INFN sezione di Bologna, Viale Berti Pichat, 6/2 - 40127 Bologna, Italy \\ ${ }^{d} I P H C$, Université de Strasbourg, CNRS/IN2P3, Strasbourg, France \\ ${ }^{e}$ INFN Laboratori Nazionali di Frascati, Via Enrico Fermi, 40 - 00044 Frascati (RM), Italy \\ ${ }^{f}$ INFN sezione di Roma, Piazzale Aldo Moro, 2 - 00185 Roma, Italy \\ ${ }^{g}$ Università di Bologna, Dipartimento di Fisica, Via Irnerio, 46 - 40126 Bologna, Italy \\ ${ }^{h}$ Università degli Studi di Milano Bicocca, Dipartimento di Fisica, Piazza della Scienza, 3 - \\ 20126 Milano, Italy \\ ${ }^{i}$ INFN sezione di Trieste, Via Valerio, 2 - 34127 Trieste, Italy
}

\begin{abstract}
The goal of the INFN SCENTT R\&D project is to develop the calorimeter technologies for the instrumentation of decay tunnels in conventional neutrino beams. This instrumentation is required to achieve a substantial improvement in the uncertainty on neutrino fluxes for the next generation cross section experiments. In particular, we are designing a positron tagger based on purely calorimetric techniques that is able to measure the rate and the spectrum of the positrons produced in the $K^{+} \rightarrow e^{+} \pi^{0} \nu_{e}$ decay. The $\nu_{e}$ flux is inferred from the positron rate in the decay tunnel. Considering the large dimensions of the tagger, the most cost effective technology is based on small modules of $\mathrm{Fe} /$ Scintillator shashlik calorimeters, with adequate segmentation and energy resolution to efficiently tag the positrons over the charged pion background. This contribution presents preliminary results obtained with two shashlik calorimeter prototypes readout with an array of Silicon PhotoMultipliers and tested at the CERN PS-T9 beamline.
\end{abstract}

Keywords: Calorimeter, Neutrino, Silicon Photomultipliers 


\section{High precision cross sections in neutrino physics}

To meet the challenges of the next generation of oscillation experiments, novel techniques must be developed to substantially reduce uncertainties on cross sections and, in general, to provide a superior characterization of the 5 artificial neutrino sources at the $\mathrm{GeV}$ scale.

At present, the main systematics affecting absolute neutrino cross section measurements are due to the knowledge of the initial neutrino flux. Since the yield of $\nu_{\mu}$ cannot be monitored by counting the number of parent pions, flux estimation mostly relies on extrapolations from hadro-production data and Monte

10 Carlo simulations. This approach limits the maximum achievable precision on the neutrino cross section to $\sim 10 \%$.

The most straightforward technique to bring flux systematics below $1 \%$ for $\nu_{e}$ cross section measurements is based on the three-body semileptonic decays of charged kaons. The $K^{+} \rightarrow e^{+} \pi^{0} \nu_{e}\left(\mathrm{~K}_{e 3}\right)$ decay offers a pure source of $\nu_{e}$ that can 15 be measured very precisely monitoring the positrons in the decay tunnel $[1,2]$. This is a standard technique used in kaon physics experiments but it has never been upscaled to the intensities required by neutrino physics due to the large rates. Cross section experiments, however, do not need an event-by-event tag of the kaons: if the $\mathrm{K}_{e 3}$ decay is the only source of $\nu_{e}$, monitoring the local 20 positron production rate along the decay tunnel is enough to lower the $\nu_{e}$ yield uncertainty to the $\%$ level.

The design of a beamline where the only source of $\nu_{e}$ is the $K_{e 3}$ decay can be obtained increasing the average momentum of the selected secondaries and reducing the length of the decay tunnel. On the other hand, the positron 25 identification against the background of charged pions and converted photons requires fast calorimeters with longitudinal segmentation. Considering that a large positron efficiency is only reached if a significant fraction of the decay tunnel is instrumented, the detector choice must be very cost-effective.

The SCENTT R\&D is focused on cheap, compact and longitudinally sampled so shashlik calorimeters readout by Silicon PhotoMultipliers (SiPMs) [3]. The 


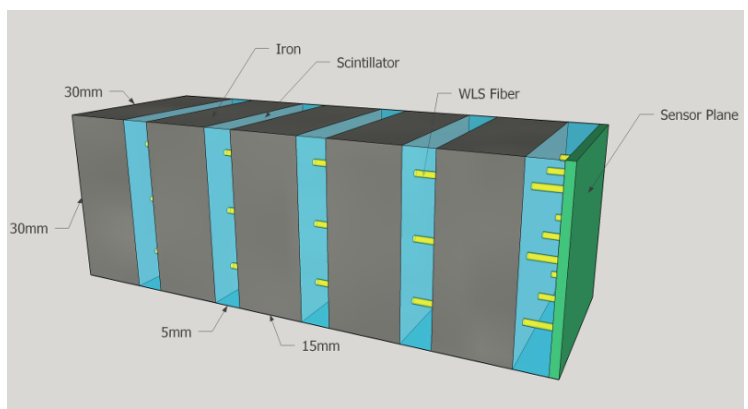

(a)

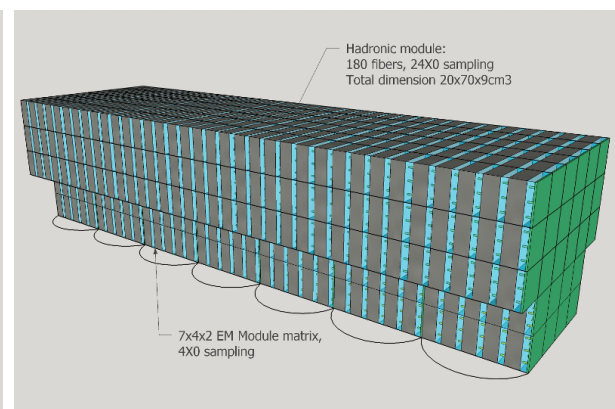

(b)

Figure 1: Scheme of the shashlik module (a) and of the complete calorimeter assembly (b) designed by the SCENTT collaboration.

baseline elements of the calorimeter are the following [2]:

- small $\left(3 \times 3 \mathrm{~cm}^{2}\right.$ area, $\left.\sim 4 X_{0}\right) \mathrm{Fe} / \mathrm{Scintillator} \mathrm{shashlik} \mathrm{modules,} \mathrm{each}$ readout by 9 wavelength shifter fibers (WLS) and 9 SiPMs (Fig. 1a);

- the small modules will be arranged in a $7 \times 2 \times 4$ matrix in order to contain the EM showers up to a few GeV (Fig. 1b);

- a larger shashlik module with a coarser longitudinal segmentation will be placed on top of the EM layers to contain the hadronic showers (for a total of $\left.3.1 \lambda_{I}\right)$;

- the readout electronics will be based on waveform digitizers to cope with the expected particle rate of $500 \mathrm{kHz} / \mathrm{cm}^{2}$.

This design poses tight constraints both to the mechanics and to the frontend electronics:

- the electronic layer where the SiPMs are hosted must be as thin as possible, in order to reduce the dead zones of the calorimeter;

45 - considering their position in the middle of the EM showers, the SiPMs must withstand a moderate radiation damage (1.3 kGy) and must be insensitive to nuclear counter effects [4]; 
- to reduce the number of channels, the signals of the 9 SiPMs of the EM modules will be summed together without amplification. The single cell gain and the intrinsic photodetection efficiency (PDE) of the SiPMs should be large enough to ensure sensitivity to MIPs;

- given the large number of channels, particular attention will be paid on the selection of the sampling frequency and resolution of the waveform digitizers, trying to reduce as much as possible the cost per channel.

This contribution describes the preliminary results in terms of energy resolution obtained with two shashlik calorimeter modules. The modules are readout using an array of SiPMs. The calorimeters have been tested at CERN on the PS-T9 beamline using a mixed beam composed of muons, pions and electrons in the $1-5 \mathrm{GeV}$ energy range.

\section{The shashlik prototypes}

The shashlik calorimeter prototypes are a modified version of the calorimeter tested by the FACTOR collaboration in 2009 [5]: they consists of two modules (from now on mod.A and mod.B) of $408 \times 8 \mathrm{~cm}^{2}, 3.3 \mathrm{~mm}$ thick tiles of lead and plastic scintillator (20/20 lead/scintillator), for a total of $\sim 12 X_{0}$ (Fig. 2).

${ }_{65}$ The light readout is performed by means of $640.8 \mathrm{~mm}$ Kuraray-Y11 WLS fibers: mod.B is longitudinally segmented by WLS fibers with different lengths (the whole calorimeter length and only the second half of it), while mod.A is equipped only with full length fibers.

The WLS fibers are collected in a plastic holder, which is used also as a mechanical support for the electronic board hosting the SiPMs. 64 SiPMs manufactured by Advansid in a plastic SMD package (model ASD-RGB1C-P, 673 $40 \times 40 \mu \mathrm{m}^{2}$ cells) are soldered on a custom PCB, directly interfaced to the calorimeter in order to minimize as much as possible the dead zones. The electronic board provides the signal routing of the SiPMs through MCX connectors and four independent biases, corresponding to four different regions of 


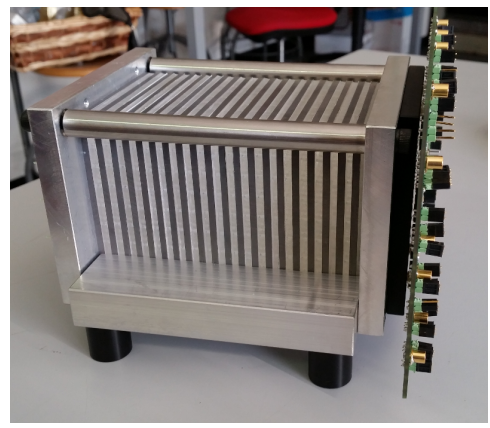

Figure 2: Picture of the calorimeter prototype.

the board: the SiPMs have been previously selected, grouping the devices with the same breakdown voltage in the same bias zone in order to provide the same overvoltage to all SiPMs with an accuracy of $0.1 \mathrm{~V}$. The $64 \mathrm{SiPMs}$ can be readout independently, or summed together in groups of four, reducing the number of readout channels to 16: the summed configuration can be selected switching the jumpers on the board itself.

\section{Experimental setup and DAQ}

The shashlik calorimeter prototypes have been tested at CERN on the PST9 beamline using a mixed beam of muons, pions and electrons up to $5 \mathrm{GeV}$.

85 The experimental setup on the T9 beamline (Fig. 3) consists of:

- one Cherenkov detector for the electron tagging;

- two silicon strip beam chambers [6] for the track reconstruction with a spatial resolution of $\sim 30 \mu \mathrm{m}$;

- a $10 \times 10 \mathrm{~cm}^{2}$ plastic scintillator for the trigger;

- the calorimeter modules, hosted inside a darkened metal box.

The DAQ system was a standard VME system controlled by a SBS Bit3 model 620 bridge $^{1}$, optically linked to a Linux PC-system. The calorimeter

\footnotetext{
${ }^{1}$ SBS Technologies Inc., US, http://www.ge-ip.com
} 


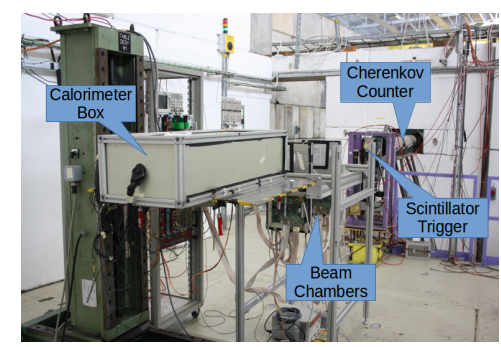

Figure 3: Picture of the experimental setup on the T9 beamline.

signal readout was performed with a charge integrating ADC (mod. V792 QDC, CAEN), delaying the signals by $120 \mathrm{~ns}$ to cope with the integration gate.

95

\section{Results}

Events are pre-selected using the information provided by the silicon strip detectors. Only single track events impinging on a $4 \times 4 \mathrm{~cm}^{2}$ fiducial area are retained. The electron events are then tagged using the information provided by the Cherenkov detector. The plots of the electrons energy deposit in the calorimeter are fitted with a Crystal Ball [7] function, whose mean and sigma values are used to compute the linearity and energy resolution. The linearity and resolution results for mod.A, obtained with a SiPM overvoltage of $5 \mathrm{~V}$, are presented in Figs. 4 and 5. The energy resolution data points are fitted with a $\frac{\sigma_{E}}{E}=\frac{S}{\sqrt{E}} \oplus C$ function, where $S$ and $C$ represent the stochastic and constant terms; more details on the physical meaning of these parameters can be found in $[8]$.

It can be noted that the output of the summed SiPM induces non-negligible saturation effects from $4 \mathrm{GeV}$. The collected signals show deviations from the linearity of the order of $2 \%$ at $4 \mathrm{GeV}$ and $8 \%$ at $5 \mathrm{GeV}$. This effect is partially due to the limited shower containment of the calorimeter (at $5 \mathrm{GeV}$ the containment of the two modules is only 83\%) and to the relative small number of available pixels in the SiPMs. This problem will be solved in the SCENTT modules with a careful selection of the SiPM dynamic range, employing SiPMs with smaller cells. The stochastic energy resolution term amounts to $\sim 13 \%$ and is 


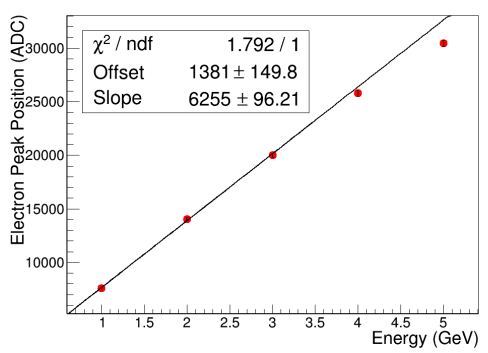

Figure 4: Calorimeter energy linearity, mod.A.

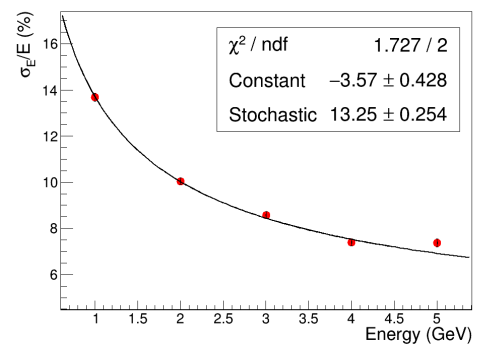

Figure 5: Calorimeter energy resolution, mod.A.

115

mostly due to the longitudinal shower leakage. The new readout scheme shows better performance than the bundling scheme employed in [5] due to the larger fiber cross sectional area covered by the SiPM.

In Fig. 6, the energy resolution of mod.B is shown as a function of the energy. The increase of the stochastic term with respect to mod.A (from $~ 13 \%$ to $\sim 16 \%$ ) is due to the non uniform sampling of the shower and to the reduced amount of collected light.

However, the light collected by short and long fibers provides information on the longitudinal development of the shower. Fig. 7 presents the signal collected by long versus short fibers: the regions populated by electrons, pions and MIPs (non showering pions and muons) are clearly visible. 


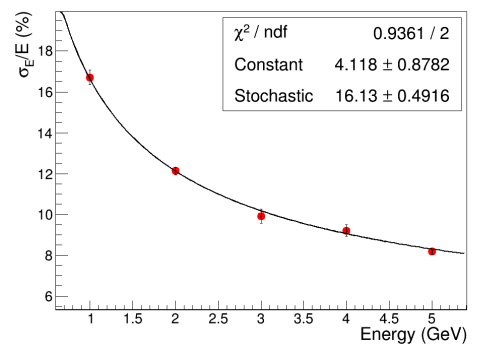

Figure 6: Calorimeter energy resolution, mod.B.

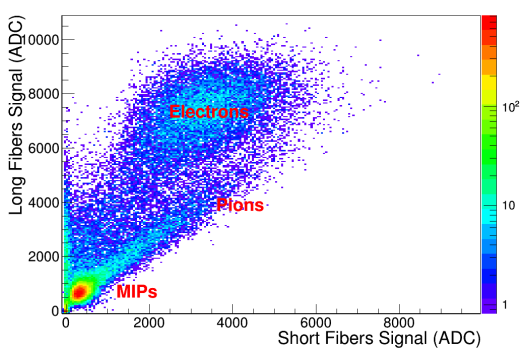

Figure 7: Scatter plot of the long and short fibers signals, mod.B.

\section{Conclusions and outlook}

Two prototypes of shashlik calorimeters with an embedded compact readout system based on SiPMs without fiber bundling have been tested on the CERN PS-T9 beamline. The prototypes have been characterized in terms of linearity and energy resolution. These tests represent the first step towards the assembly of an ultra-compact shashlik module that will be developed by the SCENTT collaboration as the basic block for large calorimeter systems. The results obtained in terms of energy resolution demonstrate that the new readout is an effective replacement for the standard systems based on fiber bundling, substantially improving the compactness of the calorimeter. The complete characterization analysis of mod.A and B is in progress [9] and two beamtests are planned in 2016 with the SCENTT modules. 


\section{Acknowledgments}

${ }_{140}$ This project has received funding from the European Unions Horizon 2020 Research and Innovation programme under Grant Agreement no. 654168. We would also like to thank the PS staff, in particular Henric Wilkens and Lau Gatignon for the support during the data taking on the PS-T9 beamline.

\section{References}

145 [1] A. Longhin, L. Ludovici and F. Terranova, Eur. Phys. J. C 75 (2015) 155;

[2] A. Berra et. al., arXiv:1512.08202 [hep-ex];

[3] The neutrino physics opportunities and the general technology for nonconventional kaon-tagged neutrino beams will be developed in the framework of the EU Horizon 2020 Project ENUBET (ERC Consolidator Grant 2015, PI: Andrea Longhin);

[4] D. Renker and E. Lorenz, Journal of Instr. 4 (2009) P04004;

[5] A. Berra et. al., IEEE Trans. Nucl. Sci. 58 (2011) 1297-1307;

[6] M. Prest et. al., Nucl. Instr. and Meth. in Phys. Res. A 501 (2003) 280-287;

[7] T. Skwarnicki, DESY F31-86-02 (1986), Ph.D Thesis, Appendix E;

[8] C. W. Fabjan and F. Gianotti, Rev. Mod. Phys. 75 (2003) 1243-1286;

[9] A. Berra et. al., "A compact light readout system for longitudinally segmented shashlik calorimeters", in preparation. 